\title{
Rapid Transport of Biomolecules along Frictionless Wavy-Rough Interfaces
}

\author{
Kwang-Hua Chu \\ School of Mathematics, Physics, and Biological Engineering, Inner Mongolia University of \\ Science and Technology, Baotou, China \\ Email: chukh49@gmail.com
}

Received February 20, 2013; revised March 25, 2013; accepted April 3, 2013

Copyright (C) 2013 Kwang-Hua Chu. This is an open access article distributed under the Creative Commons Attribution License, which permits unrestricted use, distribution, and reproduction in any medium, provided the original work is properly cited.

\begin{abstract}
There are many types of almost frictionless and very rapid flows of complex molecules in nature. One example is the almost frictionless nature of the protein channel aquaporin-1. Nearly frictionless transport of complex biomolecular fluids along a membrane composed of wavy-rough nanoannuli was investigated by using the verified Eyring's transition-state approach (cf. Journal of Physical Chemistry B, Vol. 112, 2008, pp. 3019-3023.), together with a boundary perturbation approach, which has been successfully adopted to study the selective transport of polymeric matter in confined nanodomains. The critical parameters related to the rapid or nearly frictionless transport of biomolecules were identified to be directly relevant to the possible phase-transition temperature after selecting specific activation energies and activation volumes for fixed confined (cylindrical) geometry as well as small wavy corrugations along the interface. Our results can also make the membrane composed of aligned wavy-rough nanotubes a promising mimic of protein channels for transdermal drug delivery and selective chemical sensing.
\end{abstract}

Keywords: Aquaporin; Activation; Transition State

\section{Introduction}

The discovery of water channel proteins, termed aquaporins, has revealed the molecular basis for understanding fluid absorption and secretion through cell membranes in response to even very small osmotic gradients created by solute movement [1,2]. Aquaporins form water channels that play major roles in a variety of physiological processes so that altered expression or function may underlie pathological conditions [3-5]. Note that water transport across the cell membrane is essential for the maintenance of the intracellular environment. Physiological evidence has shown that water molecules move through a selective pore. Despite some qualitative similarities in fluid behavior in cylindrical and slit-like pores, the transport of fluids in confined nanodomains is different in important details, especially for narrow pores [6]. Thus, the boundary conditions for the microscopic domain must be well prescribed!

One prominent difference between the fluid motions in microscale tubes and those in macroscale tubes is the strong fluid-wall interactions observed in microdomains and nanodomains. As the tube size decreases, the sur- face-to-volume ratio increases. Therefore, various properties of the walls or interfaces, such as surface roughness, greatly affect the fluid motions in microdomains and nanodomains. Although laminar flows have been known to be only slightly influenced by surface roughness in macroscale tubes [7], the effect of surface roughness is expected to be significant in microtubes and nanotubes. Indeed, the amplitude of surface roughness is only one or two orders of magnitude lower than the channel height for many microtubes. In this paper, we shall adopt the verified transition-state or absolute-reaction approach [8,9] which was confirmed [10] that this model developed originally by Eyring [8] can be used to describe fluid flows on the nanoscale. To consider the more realistic but complicated boundary conditions in the walls or interfaces of microdomians and nanodomains (which could be a basic element of a bio-membrane); however, we will use the boundary perturbation technique $[7,9]$ to handle the presumed (approximately) wavyroughness along the walls or interfaces of nanotubes. The relevant boundary conditions [11,12] along the wavyrough surface will be prescribed below after we introduce the Eyring's model $[8,13]$. 


\section{Theoretical Formulations}

Generally, aquaporins facilitate the movement of water across cell membranes in response to osmotic gradients, functioning in cellular and organismal osmoregulation and solute transport [14,15]. Recent high-resolution structures (via electron and X-ray crystallography) suggest that selectivity for water is accomplished by a filter that excludes larger molecules and a hydrophobic entrance to the pore that blocks the passage of hydrated ions [16-18]. Electrostatic interactions between highly conserved asparagine residues and water molecules in the pore disrupt the hydrogen bonding pattern that would be formed by the chain of water molecules, thereby preventing the conduction of protons [17]. The unusual combination of a hydrophobic pore and a small number of solute binding sites was proposed to facilitate water transport [18]. With above knowledge, we shall borrow and introduce the Eyring's approach.

Eyring derived a hyperbolic sine relation between the shear rate $(\dot{\xi})$ and the shear stress $(\tau)$ [8,13] with sound physical foundation from the thermal activation process $[8,9,10,13]$.

This polymeric fluid is associated with the momentum transfer between neighboring atomic clusters on the microscopic scale and reveals the atomic interaction in the relaxation of viscous flow. It is worth pointing out that the Eyring fluid requires the interaction between atoms in the direction perpendicular to the shearing direction for the momentum transfer. The momentum transfer depends on the activation shear volume, which is associated with the center distance between atoms and is proportional to $k_{B} T / \tau_{0} \quad\left(k_{B}\right.$ is the Boltzmann constant, $T$ is the temperature in Kelvin, and $\tau_{0}$ a constant with the dimension of stress).

We consider a steady fully developed flow of the polymeric fluid in a wavy-rough microtube of $a$ in meanaveraged radius and the outer wall being a fixed wavyrough surface, $r=a+\varepsilon \sin (k \theta)$ where $(r, \theta)$ is the cylindrical coordinate we adopt, $\varepsilon$ is (peak) amplitude of the (wavy) roughness, and $k$ is the wave number (cf. Figure 1).

Firstly, the polymeric fluid can be expressed as [8,9]

$$
\dot{\xi}=\dot{\xi}_{0} \sinh (\Pi r / a),
$$

with the forcing parameter

$$
\Pi=(-a \cdot \mathrm{d} p / \mathrm{d} z) /\left(2 \tau_{0}\right)
$$

where

$$
\dot{\xi}_{0}=C_{0} k_{B} T \exp \left(-\Delta E / k_{B} T\right) / h,
$$

with $C_{0}$ : a constant relating rate of strain to the jump frequency, accounting for the interchain co-operation required; $\Delta E$ is the activation energy, $h$ is the Planck constant, $\tau_{0}\left(=2 k_{B} T / V_{\mathrm{h}}\right)$ is functions of temperature

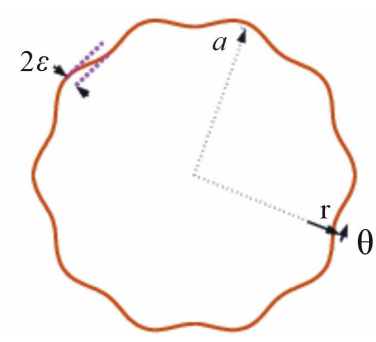

Figure 1. Schematic of a microtube with wavy-rough wall. $\varepsilon$ is the amplitude of the roughness and the wave number of wavy roughness is $k(=10)$.

and have the same dimension as $\tau ; \mathrm{d} p / \mathrm{d} z$ is the pressure-gradient along the microtube-axis direction $(z)$ (for small shear stress $\tau \ll \tau_{0}, \tau_{0} / \dot{\xi}_{0}$ represents the viscosity of the material). Note that $V_{h}=\lambda \lambda_{2} \lambda_{3}$ is the activation volume for the molecular event $[8,9]$ with $\lambda$ being the average distance between equilibrium positions in the direction of motion, $\lambda_{2}$ being the distance between neighboring molecules in this same direction (which may or may not equal $\lambda$ ), $\lambda_{3}$ being the molecule to molecule distance in the plane normal to the direction of motion (cf. Figure 2) [8].

\section{Slip Velocity}

As $\dot{\xi}=-\mathrm{d} u / \mathrm{d} r$ ( $u$ is the velocity of the fluid flow in the longitudinal ( $z$-)direction of the microtube), after integration, we obtain

$$
u=u_{s}+\dot{\xi}_{0} a[\cosh \Pi-\cosh (\Pi r / a)] / \Pi,
$$

here, $u_{s} \equiv u_{\text {slip }}$ is the velocity over the surface of the microtube [11], which is determined by the boundary condition [7]. We noticed that a general slip boundary condition for transport over a solid surface was proposed [12] as

$$
\Delta u=L_{s}^{0} \dot{\xi}\left(1-\dot{\xi} / \dot{\xi}_{c}\right)^{-1 / 2},
$$

where $\Delta u$ is the velocity jump over the boundary (solid) surface, $L_{s}^{0}$ is a constant slip length, $\dot{\xi}_{c}$ is the critical shear rate at which the slip length diverges. With the boundary condition from $[9,12]$, we shall derive the velocity field and volume flow rate along the wavy-rough microtube below using the boundary perturbation technique [7,9].

We firstly select $L_{s}^{0}$ to be the characteristic length scale and set $r^{\prime}=r / L_{s}^{0}, z^{\prime} / L_{s}^{0}, R_{0}=a / L_{s}^{0}, \varepsilon=\varepsilon / L_{s}^{0}$. After this, for simplicity, we drop all the primes. It means, from now on, $r, z, R_{0}$, and $\varepsilon$ become dimensionless. Along the boundary, we have $\dot{\xi}=\left.(\mathrm{d} u / \mathrm{d} n)\right|_{\text {on surface }}$. Here, $n$ means the normal.

Let $u$ be expanded in $\varepsilon$ :

$$
u=u_{0}+\varepsilon u_{1}+\varepsilon^{2} u_{2}+\cdots
$$

and on the boundary, we expand $u\left(R_{0}+\varepsilon \mathrm{d} r, \theta\left(=\theta_{0}\right)\right)$ into 


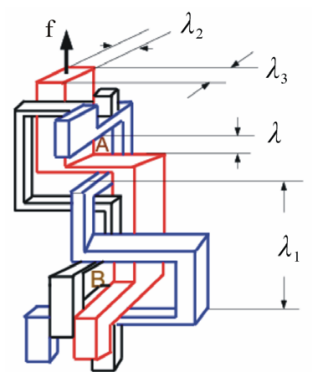

Figure 2. Schematic model of transport in a polymeric fluid (composed of composite biomolecules) $[8,13]$. Under an applied force (f) of sufficient magnitude a kink in the composite particles will overcome the activation barrier and jump to an available hole (at $A$ or B).

$$
\begin{aligned}
& \left.u(r, \theta)\right|_{\left(\mathrm{R}_{0^{+} \varepsilon \mathrm{d} r}, \theta 0\right)} \\
& =u\left(\mathrm{R}_{0}, \theta\right)+\varepsilon\left[\mathrm{d} r u_{r}(r, \theta)\right] \\
& +\varepsilon^{2}\left[u_{r r}\left(\mathrm{R}_{0}, \theta\right) \mathrm{d} r^{2} / 2\right]+\cdots \\
& =\left.\left\{u_{\text {slip }}+\dot{\xi}_{0} R_{0}\left[\cosh \Pi-\cosh \left(\Pi r / r_{2}\right)\right] / \Pi\right\}\right|_{\text {on surface }},
\end{aligned}
$$

where

$$
\left.u_{\text {slip }}\right|_{\text {on surface }}=\left.L_{s}^{0}\left[\dot{\xi}\left(1-\dot{\xi} / \dot{\xi}_{c}\right)^{-1 / 2}\right]\right|_{\text {on surface }},
$$

and $u_{r} \equiv \partial u / \partial r$.

Now, on the wall (for $\dot{\xi}$ ), we have [7,9]

$$
\begin{aligned}
& \frac{\mathrm{d} u}{\mathrm{~d} n}=\nabla u \cdot \frac{\nabla\left(r-R_{0}-\varepsilon \sin (k \theta)\right)}{\left|\nabla\left(r-R_{0}-\varepsilon \sin (k \theta)\right)\right|} \\
& =\left[1+\varepsilon^{2} \frac{k^{2}}{r^{2}} \cos ^{2}(k \theta)\right]^{-1 / 2} \\
& \quad\left[u_{r}\left|\left(R_{0}+\varepsilon \mathrm{d} r, \theta\right)-\frac{\varepsilon k}{r^{2}} \cos (k \theta) u_{\theta}\right|_{\left(R_{0}+\varepsilon \mathrm{d} r, \theta\right)}\right] \\
& =\left.u_{0_{r}}\right|_{R_{0}}+\varepsilon\left[u_{1_{r}}\left|R_{0}+u_{0_{r r}}\right|_{R_{0}} \sin (k \theta)-\left.\frac{k}{r^{2}} u_{0_{\theta}}\right|_{R_{0}} \cos (k \theta)\right] \\
& +\varepsilon^{2}\left[\left.\frac{-k^{2}}{2 r^{2}} \cos ^{2}(k \theta) u_{0_{r}}\right|_{R_{0}}+\left.u_{2_{r}}\right|_{R_{0}}+u_{1_{r r}}\left|1 / 2 u_{R_{0}} \sin (k \theta)+\right|_{R_{0}}\right. \\
& \sin ^{2}(k \theta)-\frac{k}{r^{2}} \cos (k \theta)\left(\left.u_{1_{\theta}}\right|_{R_{0}}+\left.u_{0_{\theta r}}\right|_{R_{0}} \sin (k \theta)\right)+O\left(\varepsilon^{3}\right)
\end{aligned}
$$

From above equations, we can obtain the velocity fields (up to the second order) and then we can integrate them with respect to the cross-section to get the volume flow rate $Q \equiv Q_{\text {rough }}=Q_{\text {smooth }}+\varepsilon^{2} Q_{2}$ up to the second order here.

\section{Numerical Results and Discussion}

The following figures are for our primary interest: Higher flux or lower resistance (viscosity) which means rather low resistance of the pressure-driven transport of many biomolecules. This can be understood from the net transport $Q(\propto \sinh \Pi)$. We have the average velocity: $v=$ $Q / A_{m}$ with $A_{m}$ being the effective area. After this, we then have the flux: $\rho v=|\mathbf{J}|$ which is proportional to the inverse of the flow resistance. The latter is directly relevant to the frictional resistance or shear stress along the tube wall considering the balancing of the driven pressure force when the transport is finally steady or fully developed. This can be evidenced easily once $\Pi$ is small then we have $\mathrm{v} \propto \sinh \Pi \propto \Pi \propto|\mathrm{d} p / \mathrm{d} z|$.

With the Eyring's transition-state model [8,13] (of stress-biased thermal activation), structural rearrangement is associated with a single energy barrier (height) $\Delta E$ that is lowered or raised linearly by a (shear) yield stress $\tau$. If the transition rate is proportional to the shear strain rate (with a constant ratio: $K_{0} \approx 2 V_{\mathrm{h}} / V_{\mathrm{m}}$ ), we can calculate the shear stress

$$
\tau=E / V_{h}+\left(k_{B} T / V_{h}\right) \ln \left(\dot{\xi} / K_{0} v_{0}\right),
$$

with $v_{0}$ is an attempt frequency $[8,13]$.

Figure 3 shows that when the shear strain rate $\left(\dot{\xi}_{0}\right)$ is of the order of magnitude $O\left(10^{3} \mathrm{~s}^{-1}\right)$ there are possible frictionless states for pressure driven many-biomolecule flows at the room temperature regime $(T c \sim 300 \mathrm{~K})$. Here $a=r_{2}=0.1 \mu \mathrm{m}$ and $\varepsilon=0.1$. The activation energy is $9 \times$ $10^{-20}$ Joule and the activation volume is $3.12 \times 10^{-21} \mathrm{~m}^{3}$ $(T \sim 300 \mathrm{~K})$. We can observe that there is a sharp decrease of shear stress (or resistance) starting from around $T \sim 300.5 \mathrm{~K}$. Below around $300 \mathrm{~K}$, the pressure driven transport of many biomolecules is nearly frictionless.

Possible explanation for this almost frictionless transport could be due to the tunneling as the shear thinning removes the barriers. It might be understood that the creation of a large amount of free volume (voids or

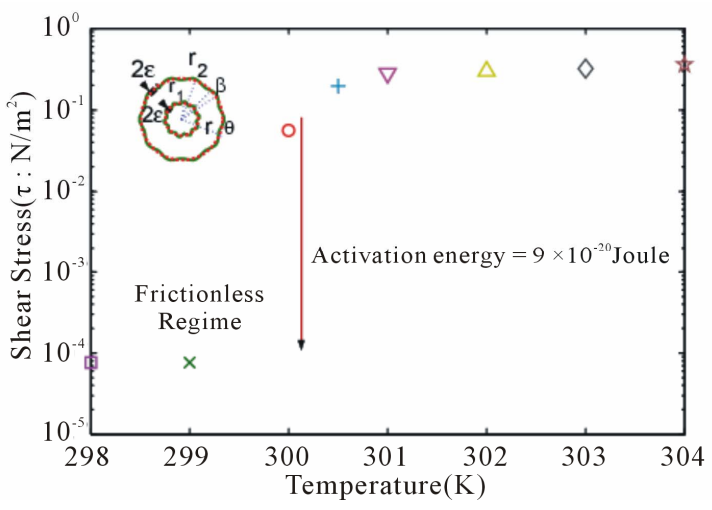

Figure 3. Calculated (shear) stresses or resistance using an activation energy $9 \times 10^{-20} \mathrm{~J}$. There is a sharp decrease of shear stress starting from around $T \sim 300 \mathrm{~K}$. Below around $300 \mathrm{~K}$, the pressure driven transport of many biomolecules is nearly frictionless where the shear strain rate $\left(\dot{\xi}_{0}\right)$ is of the order of the magnitude $O\left(10^{3} \mathrm{~s}^{-1}\right)$. 
holes) reduces the barrier for biomolecules or holes to move; i.e., it enhances the mobility of the biomolecules or holes. We remind the readers that aquaporins are members of the Major Intrinsic Protein (MIP) superfamily, found in animals, plants, insects, and bacteria [19-24]. To be specific, aquaporin-mediated water transport across cellular membranes is an ancient, ubiquitous mechanism within cell biology. This family of integral membrane proteins includes both water selective pores (aquaporins) and transport facilitators of other small molecules such as glycerol and urea (aquaglyceroporins).

Eukaryotic aquaporins are frequently regulated posttranslationally by gating, whereby the rate of flux through the channel is controlled, or by trafficking, whereby aquaporins are shuttled from intracellular storage sites to the plasma membrane [25]. With present approach and numerical calculations, we can apply them to the diverse fields of researches related to aquaporins or MIPs in the future [26-29].

\section{Conclusion}

In brief summary, we have numerically obtained the possible rapid transport (up to the second order) inside the wavy-rough microconduits by using the boundary perturbation method and demonstrated the possible very-low resistance state via the tuning of the activation energy and volume at room temperature regime. Our theoretical results show that for smaller forcing (along the microtube-axis direction; relative to the referenced shear stress), there are significantly different flow rates for different amplitudes of wavy-rough microconduits. The latter result could be directly applied to the efficient biomolecular sieving and separation. Our results should be useful to the design of microfiltration devices (using microtube networks and assemblies), flow control in microfluidics, and other similar applications in biophysics and biochemistry researches. Our next goal is to design and achieve a generic molecular sieving structure for an integrated biomolecule sample preparation and analysis system.

\section{Acknowledgements}

The only author would like to thank the partial support of 2013-Inner Mongolia University of Science and Technology_Starting Funds for Scientific Researcher. The only author will be at: 2/F, 24, Lane 260, Section 1, Road Muja, Taipei, Taiwan 116, China after 2013-June.

\section{REFERENCES}

[1] M. Majumder, N. Chopra, R. Andrews and B. J. Hinds, "Nanoscale Hydrodynamics-Enhanced Flow in Carbon Nanotubes," Nature (London), Vol. 438, No. 44, 2005, p. 930. doi:10.1038/438930b
[2] G. Hummer, J. C. Rasaiah and J. P. Noworyta, "Water Conduction through the Hydrophobic Channel of a Carbon Nanotube," Nature (London), Vol. 414, 2001, pp. 188-190. doi:10.1038/35102535

[3] P. R. Waggoner and H. Maisel, "Immunofluorescent Study of a Chick Lens Fiber Cell Membrane Polypeptide," Experimental Eye Research, Vol. 27, No. 2, 1978, pp. 151-157. doi:10.1016/0014-4835(78)90085-4

[4] M. B. Gorin, S. B. Yancey, J. Cline, J. P. Revel and J. Horwitz, "The Major Intrinsic Protein (MIP) of the Bovine Lens Fiber Membrane: Characterization and Structure Based on cDNA Cloning," Cell, Vol. 39, No. 1, 1984, pp. 49-59. doi:10.1016/0092-8674(84)90190-9

[5] M. L. Zeidel, S. V. Ambudkar, B. L. Smith and P. Agre, "Reconstitution of Functional Water Channels in Liposomes Containing Purified Red-Cell CHIP28 Protein," Biochemistry, Vol. 31, No. 33, 1992, pp. 7436-7440. doi:10.1021/bi00148a002

[6] K. Balasubramanian and M. Burghard, "Chemically Functionalized Carbon Nanotubes," Small, Vol. 1, No. 2, 2005, pp. 180-186. doi:10.1002/smll.200400118

[7] W. K.-H. Chu, "Stokes Slip Flows between Corrugated Walls," Zeitschrift für Angewandte Mathematik und Physik, Vol. 47, No. 4, 1996, pp. 591-599. doi:10.1007/BF00914873

[8] F. H. Johnson, H. Eyring and B. J. Stover, "The Theory of Rate Processes in Biology and Medicine," Wiley, New York, 1974.

[9] K.-H. W. Chu, "Sieving Transport within Wavy-Rough Nanoannuli," Journal of Physical Chemistry B, Vol. 112, No. 10, 2008, pp. 3019-3023. doi:10.1021/jp076114f

[10] J. K. Holt, "Methods for Probing Water at the Nanoscale," Microfluidics and Nanofluidics, Vol. 5, 2008, pp. 425-442.

[11] C. L. M. H. Navier, "Mémoire sur les lois du Mouvement des Fluides," Mémoires de l'Académie Royale des Sciences de l'Institut de France, 1823, pp. 389-440.

[12] P. A. Thompson and S. M. Troian, "A General Boundary Condition for Liquid Flow at Solid Surfaces," Nature (London), Vol. 389, 1997, pp. 360-363. doi: $10.1038 / 39475$

[13] R. H. Ewell and H. Eyring, "Theory of the Viscosity of Liquids as a Function of Temperature and Pressure," Journal of Chemical Physics, Vol. 5, No. 9, 1937, p. 726. doi:10.1063/1.1750108

[14] M. J. Daniels and M. Yeager, "Phosphorylation of Aquaporin PvTIP3;1 Defined by Mass Spectrometry and Molecular Modeling," Biochemistry, Vol. 44, No. 44, 2005, pp. 14443-14454. doi:10.1021/bi050565d

[15] G. M. Preston, B. L. Smith, M. L. Zeidel, J. J. Moulds and P. Agre, "Mutations in Aquaporin-1 in Phenotypically Normal Humans without Functional CHIP Water Channels," Science, Vol. 256, No. 5055, 1992, pp. 385387. doi:10.1126/science.256.5055.385

[16] D. Fu, A. Libson, L. J. W. Miercke, C. Weitzman, P. Nollert, J. Krucinski and R. M. Stroud, "Structure of a Glycerolconducting Channel and the Basis for Its Selectivity," Science, Vol. 290, No. 5491, 2000, pp. 481-486. 
doi:10.1126/science.290.5491.481

[17] K. Murata, K. Mitsuoka, T. Hirai, T. Walz, P. Agre, J. B. Heymann, A. Engel and Y. Fujiyoshi, "Structural Determinants of Water Permeation through Aquaporin-1," $\mathrm{Na}$ ture, Vol. 407, 2000, pp. 599-605. doi:10.1038/35036519

[18] H. Sui, B.-G. Han, J. K. Lee, P. Walian and B. K. Jap, "Structural Basis of Water-Specific Transport through the AQP1 Water Channel," Nature, Vol. 414, 2001, pp. 872877. doi: $10.1038 / 414872 \mathrm{a}$

[19] G. M. Pao, L. F. Wu, K. D. Johnson, H. Höfte, M. J. Chrispeels, G. Sweet, N. N. Sandal and M. H. Saier, "Evolution of the MIP Family of Integral Membrane Transport Proteins," Molecular Microbiology, Vol. 5, No. 1, 1991, pp. 33-37. doi:10.1111/j.1365-2958.1991.tb01823.x

[20] F. Le Cahérec, S. Deschamps, C. Delamarche, I. Pellerin, G. Bonnec, M. T. Guillam, D. Thomas, J. Gouranton and J. F. Hubert, "Molecular Cloning and Characterization of an Insect Aquaporin: Functional Comparison with Aquaporin 1," European Journal of Biochemistry, Vol. 241, No. 3, 1996, pp. 707-715. doi:10.1111/j.1432-1033.1996.00707.x

[21] J. H. Park and M. H. Saier, "Phylogenetic Characterization of the MIP Family of Transmembrane Channel Proteins," Journal of Membrane Biology, Vol. 153, No. 3, 1996, pp. 171-180. doi:10.1007/s002329900120

[22] P. Agre, S. Sasaki and M. J. Chrispeels, "Aquaporins: A Family of Water Channel proteins," American Journal of Physiology, Vol. 265, 1993, p. F461.

[23] M. J. Chrispeels and P. Agre, "Aquaporins: Water Channel Proteins of Plant and Animal Cells," Trends in Biochemical Sciences, Vol. 19, No. 10, 1993, pp. 421-425. doi:10.1016/0968-0004(94)90091-4

[24] C. Maurel, “Aquaporins and Water Permeability of Plant
Membranes," Annual Review of Plant Physiology and Plant Molecular Biology, Vol. 48, 1997, pp. 399-429. doi:10.1146/annurev.arplant.48.1.399

[25] S. Törnroth-Horsefield, K. Hedfalk, G. Fischer, K. Lindkvist-Peterssonb and R. Neutze, "Structural Insights into Eukaryotic Aquaporin Regulation," FEBS Letters, Vol. 584, No. 12, 2010, pp. 2580-2588. doi:10.1016/j.febslet.2010.04.037

[26] R. Chaube, F. Chauvigné, A. Tingaud-Sequeira, K. P. Joy, A. Acharjee, V. Singh and J. Cerdá, "Molecular and Functional Characterization of Catfish (Heteropneustes fossilis) Aquaporin-1b: Changes in Expression during Ovarian Development and Hormone-Induced Follicular Maturation," General and Comparative Endocrinology, Vol. 170, No. 1, 2011, pp. 162-171. doi:10.1016/j.ygcen.2010.10.002

[27] M. Kaakinen, S. Zelenin and K. Metsikkö, “Aquaporin-4 Water Channel Oligomers Are Associated with the Transverse Tubules of Skeletal Myofibers," Experimental Cell Research, Vol. 317, No. 1, 2011, pp. 20-28. doi:10.1016/j.yexcr.2010.09.004

[28] Y. Hirano, N. Okimoto, I. Kadohira, M. Suematsu, K. Yasuoka and M. Yasui, "Molecular Mechanisms of How Mercury Inhibits Water. Permeation through Aquaporin-1: Understanding by Molecular Dynamics Simulation," Biophysical Journal, Vol. 98, No. 8, 2010, pp. 1512-1519. doi:10.1016/j.bpj.2009.12.4310

[29] B. X. Yang, A. N. van Hoek and A. S. Verkman, "Very High Single Channel Water Permeability of Aquaporin-4 in Baculovirus-Infected. Insect Cells and Liposomes Reconstituted with Purified Aquaporin-4," Biochemistry, Vol. 36, No. 24, 1997, pp. 7625-7632. doi:10.1021/bi970231r 